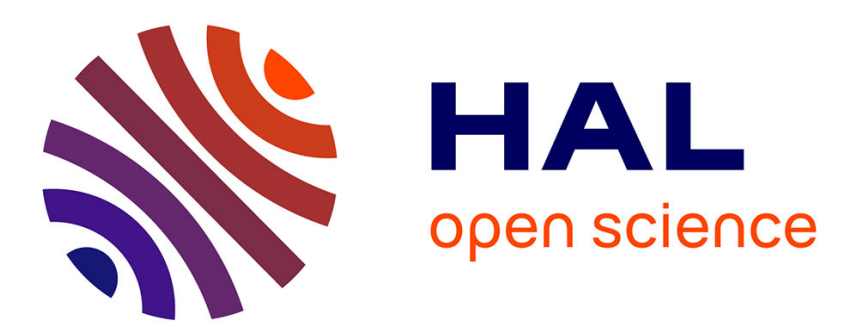

\title{
An Automated Dynamic Offset for Network Selection in Heterogeneous Networks
}

\author{
Majed Haddad, Piotr Wiecek, Habib Sidi, Eitan Altman
}

\section{To cite this version:}

Majed Haddad, Piotr Wiecek, Habib Sidi, Eitan Altman. An Automated Dynamic Offset for Network Selection in Heterogeneous Networks. IEEE Transactions on Mobile Computing, 2015, 10.1109/TMC.2015.2492560 . hal-01211210

\author{
HAL Id: hal-01211210 \\ https://hal.inria.fr/hal-01211210
}

Submitted on 4 Oct 2015

HAL is a multi-disciplinary open access archive for the deposit and dissemination of scientific research documents, whether they are published or not. The documents may come from teaching and research institutions in France or abroad, or from public or private research centers.
L'archive ouverte pluridisciplinaire HAL, est destinée au dépôt et à la diffusion de documents scientifiques de niveau recherche, publiés ou non, émanant des établissements d'enseignement et de recherche français ou étrangers, des laboratoires publics ou privés. 


\title{
An Automated Dynamic Offset for Network Selection in Heterogeneous Networks
}

\author{
Majed Haddad, member, IEEE, Piotr Więcek, member, IEEE, Habib Sidi member, IEEE \\ and Eitan Altman, Fellow, IEEE
}

\begin{abstract}
Complementing traditional cellular networks with the option of integrated small cells and WiFi access points can be used to further boost the overall traffic capacity and service level. Small cells along with WiFi access points are projected to carry over $60 \%$ of all the global data traffic by 2015 . With the integration of small cells on the radio access network levels, there is a focus on providing operators with more control over small cell selection while reducing the feedback burden. Altogether, these issues motivate the need for innovative distributed and autonomous association policies that operate on each user under the network operator's control, utilizing only partial information, yet achieving nearoptimal solutions for the network. In this paper, we propose a load-aware network selection approach applied to automated dynamic offset in heterogeneous networks (HetNets). In particular, we investigate the properties of a hierarchical (Stackelberg) Bayesian game framework, in which the macro cell dynamically chooses the offset about the state of the channel in order to guide users to perform intelligent network selection decisions between macro cell and small cell networks. We derive analytically the utility related to the channel quality perceived by users to obtain the equilibria, and compare it to the fully centralized (optimal), the full channel state information and the non-cooperative (autonomous) models. Building upon these results, we effectively address the problem of how to intelligently configure a dynamic offset which optimizes network's global utility while users maximize their individual utilities. One of the technical contributions of the paper lies in obtaining explicit characterizations of the dynamic offset at the equilibrium and the related performances in terms of the price of anarchy. Interestingly, it turns out that the complexity of the algorithm for finding the dynamic offset of the Stackelberg model is $\mathcal{O}\left(n^{4}\right)$ (where $n$ is the number of users). It is shown that the proposed hierarchical mechanism keeps the price of anarchy almost equal to 1 even for a low number of users, and remains bounded above by the non-cooperative model.
\end{abstract}

Index Terms-Heterogeneous network, macro cell, small cell, WiFi, network selection, dynamic offset, traffic steering, channel distribution information, channel state information, game theory, price of anarchy.

\section{INTRODUCTION}

To cope with the rapidly increasing demand for mobile data driven largely by mobile video and personal communications, low power base stations (BSs) are being deployed in dense urban areas to complement the existing cellular architecture [1], [2]. Such a HetNet consists of macro

Majed Haddad is with University of Avignon, France. Piotr Więcek is with Wroclaw University of Technology, Poland. Habib Sidi is with INRIA, Paris, France. Eitan Altman is with INRIA Sophia-Antipolis, France.

This research was supported by Grant S40043/K1101 of Wroclaw University of Technology. cells (MCs) coexisting with low power nodes composed of small cells (SCs), as well as using WiFi access points (APs) in unlicensed spectrum [3]. This will allow for better overall radio resource management, provide improved overall mobile-broadband performance, and allow operators to maintain a more seamless user experience [4]. Indeed, densification of the network with SCs is a way to get more capacity from existing spectrum. This results in shifting the spectrum equation as the comparison between spectrum costs and SC costs has changed dramatically. Both engineers and academics are still working out ways for SCs and WiFi APs to integrate seamlessly with macro BSs and with one another. Network access selection is a major concern. Clearly, the selection based on highest signal strength is inadequate to address this challenge. Though traditionally considered unsuitable for cellular networks, one approach is to distribute the network selection decisions over the users, as the device is the only entity aware of actual connectivity conditions (e.g., radio conditions, throughput over existing connectivity, etc.), and real-time conditions in the device (e.g., type of pending traffic, status of device including battery levels, etc.). Alternately, some regulators have proposed delegating users more involvement on the selection decision, but with retaining to the traditional network-centric selection approach. Putting aside the technological challenges that such approaches bring in many practical scenarios, it is very important that the impacts and the performances of any proposed policy are theoretically investigated.

\section{A. Controlled Network Access Selection}

SC access selection (or association) currently depends very much on the device implementation. This means that in typical implementations, the device selects the SC when it is available. Different devices may also have different implementations, leading to different user experiences. From the operator's point of view, it would be good to have more control on the access selection to be able to provide a more uniform experience. In fact, with the integration of SCs on the radio access network (RAN) levels there is a focus on providing operators with more control over $\mathrm{SC}$ access selection. This control may be gained through network-centric mobility mechanisms (e.g., direct handover command or redirection to the SC), or by device-centric mechanisms (e.g., more careful specification of the accessselection algorithm in the mobile user). The association 


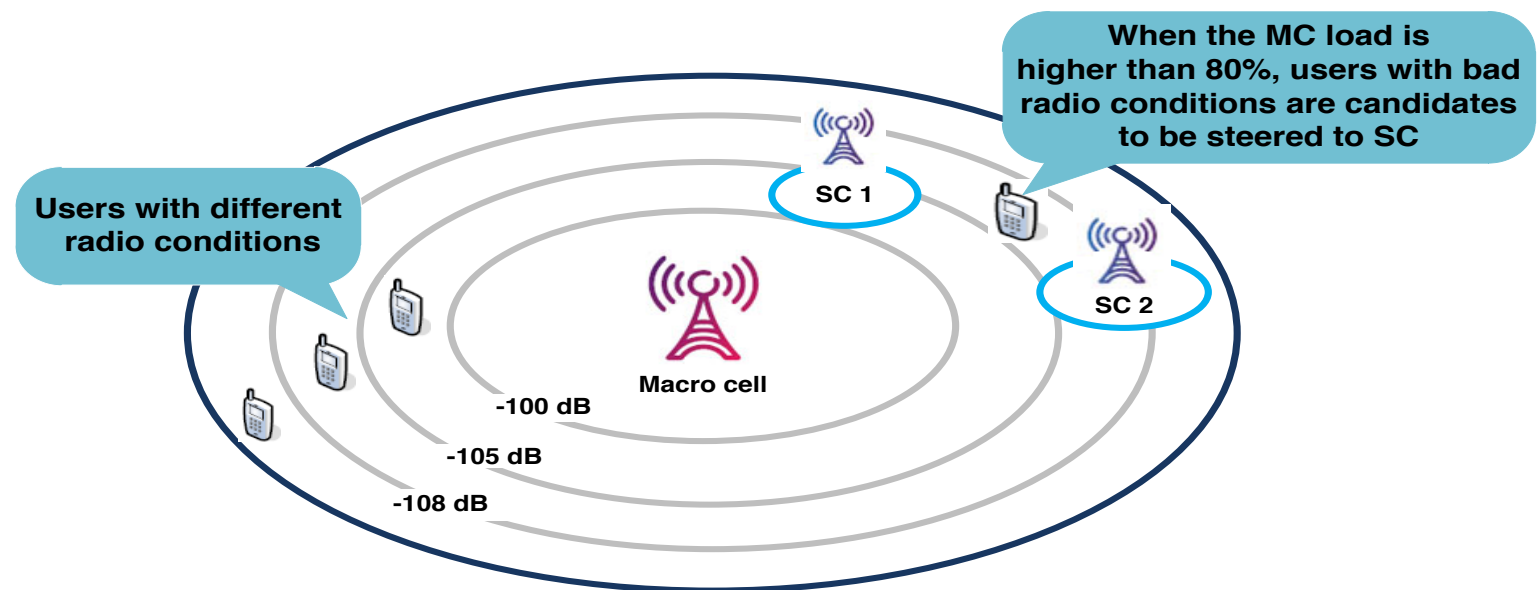

Fig. 1. Exemplary traffic steering to small cell in a heterogeneous network: Both real-time network load and radio conditions are important factors in making optimal network selection decisions.

schemes actually implemented by network operators are fully centralized: the operator tries to maximize his utility (revenue) by assigning the mobile users to different systems (for a survey refer to [5]). However, distributed joint radio resource management (JRRM) mechanisms are gaining in importance: mobile users may be allowed to make autonomous decisions in a distributed way. In the same trend, [6] proposed a distributed algorithm which jointly determine the amount of radio resources that MCs should offer to $\mathrm{SCs}$, and the association rules that decide which mobile users should associate with SCs. In [7], a network interface selection algorithm which maps user traffic across WiFi and LTE is implemented to optimize user QoE through adaptive traffic offloading. Evaluations on a real LTE-WiFi testbed using YouTube traffic reveals that video stalls are reduced by $3-4$ times compared to naive solutions. In [8], a network selection strategy considering small cell backhaul capacity is proposed and evaluated in terms of connection throughput and fairness. This has lead to game theoretic approaches to the network selection problems in HetNets, as can be found in [9]-[11]. [12] formulates the problem of user association in the downlink of small cell networks as a many-to-one matching game, in which base stations are assumed to be aware of each user's overall preferences.

Instead of taking part within the debate among the supporters of each (centralized or distributed) solution, we propose in this paper a Stackelberg formulation of the network selection problem, where the operator optimizes its global utility by sending appropriate information, while mobile users compete to maximize their throughput by picking the best locally serving RAN. By Stackelberg we mean distributed decision making assisted by the network, where the wireless users aim at maximizing their own utility, guided by aggregated information broadcasted by the network about the channel distribution information (CDI) of each user. We derive the policy that corresponds to the Stackelberg equilibrium and compare it to the fully centralized (optimal), the full channel state information (CSI) and the non-cooperative (autonomous) models.

\section{B. Partial Information}

In order to provide optimal use of RAN resources and best end user experience, 3GPP is also working on the integration of SC to include radio access technology (RAT) selection, addressed through per user and real time based decisions (e.g., network load and users' radio conditions). For example, in Fig. 1, when the cellular network is somewhat congested, the operator who controls both the MC and the SC networks may want to steer mobile users that experience bad radio conditions on the cellular network (e.g., poor Received Signal Reference Power (RSRP) ${ }^{1}$ for LTE) from the MC to the SC (assuming SCs are available). Most existing work so far have assumed that each BS or mobile user has all others' dynamics information whenever making its resource allocation decisions [13]-[16]. Nevertheless, this might not be realistic in HetNets due to the limited capacity of the backhaul links and varied ownership of network devices [17], [18]. Moreover, obtaining/estimating all the channel gains on each of the resource blocks is highly impractical, since it leads to an enormous amount of control overheads. In order to find a desired trade-off between the global network performance reached at the equilibrium and the amount of signaling needed to make it work, we investigate a strategy based on an automated dynamic offset selection where only a partial CDI is assumed to be known at the transmitter. We define the offset as the channel quality indicator (CQI) which represents a measure of the channel quality. This is a challenging network selection problem, whose solution is critical for many applications and use-cases in future wireless systems. Indeed, it has been shown that aggressively offloading mobile users from MC to SCs can lead to degradation of user-specific as well as network wide performance. On the other hand, a conservative approach may result in load disparity, which not only leads to underutilization of resources but also degrades the performance of multimedia applications [19].

\footnotetext{
${ }^{1}$ RSRP is a measure of the received signal strength of a cell at a mobile user and it is measured based on the strength of certain reference signals that cells broadcast.
} 
Clearly, in such cases any offloading strategy agnostic to these conditions is undesirable, which emphasizes the importance of more adaptive offloading/onloading strategies [20]-[24].

\section{Our Contributions}

In LTE networks, user equipments (UEs) (referred to hereafter and interchangeably, as (mobile) users or players) associate typically with the cell with highest RSRP. Recently, the introduction of enhanced inter cell interference coordination (eICIC) standards allowed the received signal strength based user association in LTE to be biased towards low power nodes by a suitable margin. The details of how much to bias the association in favor of low power nodes is left unspecified.

Our main result within this paper is to propose an alternative solution for the network operator to maximize its revenue by adequately choosing the bias (which we refer to as dynamic offset) about channel quality indicator rather than increasing the throughput (which is costly and energy inefficient). Technically, our approach not only aims at improving the network equilibrium efficiency but has also at least two nice features:

- It allows the network to guide users to a desired equilibrium that optimizes its own utility if it chooses the adequate dynamic offset to send,

- Only the individual user demand and a partial CDI of the other users is needed at each transmitter.

Automated dynamic offset for network selection problem was also considered in [25] and [24]. The major differences between these previous works and this work are: In [25], we studied the case of two users attempting to connect to Internet, having the choice between $3 \mathrm{G}$ LTE and $\mathrm{WiFi}$ networks, where the $3 \mathrm{G}$ LTE network throughput is assumed to be constant and no access control was considered at the network level. Then, in [24], we analyzed a Stackelberg formulation of the network selection problem with an arbitrary number of competing users, but without counting for the network load.

This paper considers load-aware network selection solutions, in which partial asymmetric information is available. Partial because the information available in making optimal network selection decisions is incomplete, and asymmetric because the information at the MC depends on the load, whereas the information available at the SC depends on the radio conditions. This contributes to the design of automated dynamic offset which optimizes network's global utility while intelligence is split between the MC and mobile users. We further provide a procedure on how to compute the dynamic offset for different scenarios. Interestingly, it turns out that the complexity of the algorithm for finding the dynamic offset of the Stackelberg model is polynomial in the number of users. We also obtain explicit characterizations of the dynamic offset at the equilibrium and the price of anarchy
$(\mathrm{PoA})^{2}$ of such a system. In particular, it is noteworthy to mention that when the network goal is orthogonal to users' goal, this can lead the users to a misleading association policy. The reason behind this is that the information given to the user is misleading, since the utility of the user cannot be directly inferred from the quality of his channel when the MC goal is orthogonal to users' goal.

\section{SYSTEM MODEL}

Consider a HetNet consisting of a single $\mathrm{MC}$ and a set of partly overlaying SCs operating on non-overlapping frequency bands, delivering an interference free spectrum assignment. We also assume open access control mechanism, i.e., all customers of the operator's network have the right to make use of any of the available SCs. Thus, each user arriving in the system will decide individually to which of the available systems it is best to connect according to its radio condition, its demand and the statistical information about other users. Their strategies are then based on this (incomplete) information. The association problem is then generalized to allow the MC to control the users' behavior by broadcasting appropriate information, expected to maximize its utility while individual users maximize their own utilities. $b_{i}$ is the demand of user $i . b_{i}=1$ when there exists a demand and is equal to 0 otherwise. $a_{i}$ is the action of user $i$ defined by the user's decision to connect to a certain RAN. $a_{i}=1$ when the user chooses the SC network, and $a_{i}=0$ when the user chooses the MC. $h_{i}$ is the uplink channel gain between the SC and the user. We statistically model the signal amplitude $\sqrt{h_{i}}$ as a random variable with a Rayleigh distribution. This Rayleigh fading assumption is not crucial to our work, but it makes exposition easier. By the transformation theorem for single random variable, it is well known that the effect of Rayleigh fading on power attenuation is equivalent to considering the channel gain $h_{i}$ as an exponentially distributed random variable with mean $\lambda_{i}$ [26]. We will see later how $\lambda_{i}$ is related to different parameters adopted throughout the paper. We further consider a low time-varying network in which the propagation channels maintain constant for a reasonable time interval in order for the network to converge to a stable solution.

We assume that the user state is defined by the pair $\left(h_{i}, b_{i}\right)$. The network is fully characterized by the user state. However, when distributing the JRRM decisions, this complete information is not available to the users. The MC broadcasts to UEs an aggregated information indicating a measurement of the communication quality of the wireless channel (excellent, fair, poor...). This can be done through the CQI which can be a value (or values) representing a measure of channel quality for a given channel. Typically, a high value CQI is indicative of a channel with high quality

\footnotetext{
${ }^{2}$ The PoA is the ratio of the utility attained when a network planner chooses policies to maximize social welfare versus the utility attained in $\mathrm{Nash} /$ Stackelberg equilibrium when users choose their policies strategically.
} 


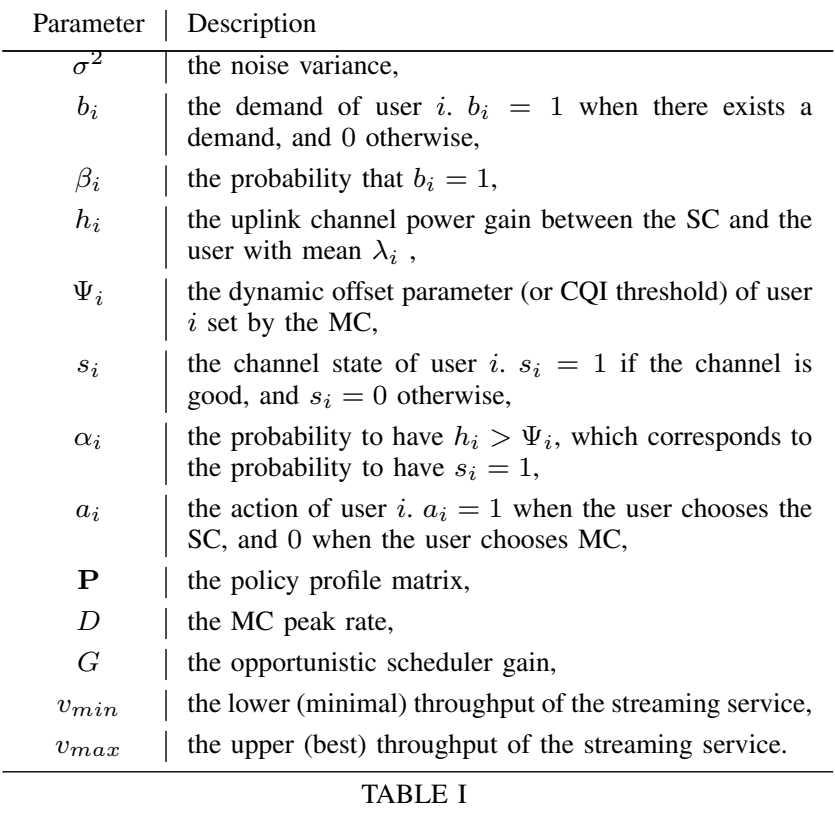

DESCRIPTION OF THE VARIOUS PARAMETERS

and vice versa. More formally, assume that the knowledge of each user about his own state is limited to the pair $\left(s_{i}, b_{i}\right)$, where $s_{i}=\mathbb{I}_{\left\{h_{i}>\Psi_{i}\right\}}$, with $\Psi_{i}$ - a fixed threshold, and $\mathbb{I}_{C}$ is the indicator function equal to 1 if condition $C$ is satisfied and to 0 otherwise. We will call $\Psi_{i}$ the $C Q I$ threshold of user $i$, referred to hereafter and interchangeably as dynamic offset. Thus, a user only knows whether he wants to transmit and whether the channel is in a good condition $\left(s_{i}=1\right)$ or in a bad condition $\left(s_{i}=0\right)$ given the CQI threshold. In addition, any player has the information about the probability distribution of his own state $\left(s_{i}, b_{i}\right)$ and that of his opponent $\left(s_{j}, b_{j}\right)$. These are given by $\alpha_{i}-$ the probability to have $h_{i}>\Psi_{i}$, and $\beta_{i}$ - the probability that the demand $b_{i}=1$. Let us denote by $\mathbf{P}=\left[\mathbf{P}_{\mathbf{1}}, \ldots, \mathbf{P}_{\mathbf{n}}\right]^{T}$ the $(n \times 2)$ policy profile matrix, whose element $\mathbf{P}_{\mathbf{i}}$ represents the action vector taken by the mobile user $i$ in low and high channel states for $i=\{1, \ldots, n\}$. The various parameters used throughout the paper are listed in Table I.

In the next sections, we provide a thorough analysis of the existence and characterization of the Bayes equilibria for both non-cooperative and Stackelberg scenarios. We first focus on the two-user case in order to gain insights into how to design decision problem in mobile wireless environments. Then, we generalize our approach to the multi-user case.

The first step before analyzing the Stackelberg Bayesian decision scheme is to define the utilities of the users. These are often related to throughput, whose variations are mainly due to network load and radio conditions.

\section{A. The Macro Cell Throughput}

When performing network selection decisions, the mobile can benefit from knowledge about real time network conditions and quality parameters. In addition, the cellular network congestion condition need to be considered. This allows the UE to perform access decisions not only based on the viability of the SC network, but also on the conditions on the MC access to determine the need to move traffic to
SC and when such traffic can indeed be moved back to the MC network.

Motivated by the fact that the exponential growth in mobile traffic is primarily driven by streaming service, we consider a real-time transport protocol (RTP) streaming service which takes into account both the cellular network congestion and the mobile user's radio conditions. The UE's utility is expressed by the quality of his streaming flow, which is in turn closely related to his throughput. Indeed, a streaming call with a higher throughput will enable the UE to stream the video at a higher bit rate and therefore offering a better video quality. The goal of a streaming user is thus to achieve the best throughput of between an upper (best) $v_{\max }$ and a lower (minimal) $v_{\min }$ bounds (with $\left.v_{\max }>v_{\min }\right)$. This throughput depends not only on the peak throughput, but also on the evolution of the number of calls in the system where the user decides to connect. Note that a user that cannot be offered this minimal throughput in neither of the available systems is blocked in order to preserve the overall network performance. However, once connected, we suppose that a call will not be dropped even if its radio conditions degrade because of mobility.

Assuming fair scheduling among different users, the throughput of a user connected to the MC when there are $n_{0}$ users admitted to $\mathrm{MC}$ is given by:

$$
v=\min \left[\frac{D \cdot G}{\sum_{i=1}^{n_{0}}\left(1-a_{i}\right) b_{i}}, v_{\max }\right]
$$

where $G$ is the opportunistic scheduler gain and $D$ is the MC peak rate (i.e., the throughput a user would obtain if he were alone in the cell). Here, we assume that the $\mathrm{MC}$ has sufficient resources to guarantee to every user the throughput he requires depending on his demand and the system load. Note that the admission control will ensure that $v \geq v_{\min }$ by blocking new arrivals. Without loss of generality, we may assume also that $D G \geq v_{\max }$.

\section{B. The Small Cell Throughput}

The measurement of average throughput of a node in a SC network is done by the time it takes to transfer the files between the SC and the mobile users. The throughput depends on the bit rate at which the wireless mobile communicates to its SC, which greatly depends on the attenuation level at the receiver side due to his geographical position. This can vary greatly depending on the link conditions. The SC throughput can thus be expressed by

$$
\log _{2}\left(1+\frac{p h_{i} a_{i} b_{i}}{\sigma^{2}}\right)
$$

where $\sigma^{2}$ is the noise variance and $p$ the user's transmit power. Notice that, in (2), we consider that there is no resource partitioning (and thus no interference) between the $\mathrm{MC}$ and the SC as they both operate on non-overlapping frequency bands.

The utility we have considered in (2) is better suited for lightly loaded SCs or for situations where each user 
is only given a tiny fraction of the SC resources regardless of the load assuming that this fraction is small enough that the SC can support all users [23]. Of course, this comes at the expense of decreasing the spectral efficiency of the network. However, we shall notice that there exists a plenty of scenarios where such a situation is met, notably situations with priority among users. For example, when resource allocation rules correspond to those of primary users in cognitive radio or spectrum pooling networks, in which each user enjoys only a limited access to the spectrum. The rationale behind this stems from the fact that primary users maximizing their own spectral efficiency independently of the others can result in suboptimal usage of the spectrum [27].

Remark 1. The BS depends crucially on truthful reporting of their channel states by the UEs. For example, in the frequency-division duplex (FDD) system, the BS has no direct information on the channel gains, but transmits downlink pilots, and relies on the UEs' reported values of gains on these pilots for scheduling. A cooperative UE will truthfully report this information to the BS. A noncooperative UE will however send a signal that is likely to induce the scheduler to behave in a manner beneficial to the UE. In our case and since we consider the uplink scenario, users do not distribute anything except their network preference list (see Fig. 2), which are obviously distributed honestly.

Given $\lambda_{i}$ and $\Psi_{i}$, we can compute that the distribution of $h_{i}$ is $\operatorname{Exp}\left(\lambda_{i}\right)$ with

$$
\alpha_{i}=\operatorname{Pr}\left\{h_{i}>\Psi_{i}\right\}=\exp \left(-\lambda_{i} \Psi_{i}\right)
$$

Knowing the information that a player has, there are four possible policies of a player $i$ with $b_{i}=1$ (we do not consider state $b_{i}=0$, when there is no transmission of any type):

\begin{tabular}{l||l|l|l|l}
$h_{i}<\Psi_{i}$ & $S$ & $S$ & $M$ & $M$ \\
\hline$h_{i}>\Psi_{i}$ & $S$ & $M$ & $S$ & $M$
\end{tabular}

where indices $M$ and $S$ stand for the MC (i.e., $a_{i}=0$ ) and the $\mathrm{SC}$ (i.e., $a_{i}=1$ ) respectively. Notice that the top row corresponds to $s_{i}=0$ while the bottom row corresponds to $s_{i}=1$. In the sequel, we will refer to these four policies shortly as $S S, S M, M S$ and $M M$ policies. Let us not consider the policy $S M$, which is irrational, as the throughput of a player using the SC when $h_{i}>\Psi_{i}$ is certainly higher than that when $h_{i}<\Psi_{i}$. We then have a game with partial CDI with two states and 3 actions for each player in every state.

\section{THE TWO-USER CASE}

For the ease of comprehension, we will begin by considering the two-user case and then generalize the results to the multi-user case later in Section IV.

\section{A. Utilities}

User $i$ 's utility in state $s=0,1$ is then given by

$u_{i}(s, \mathbf{P})= \begin{cases}v_{\mathbf{P}_{\mathrm{j}}}^{i} ; & \text { if user } i \text { chooses } M \text { at state } s, \\ C^{i}(s) ; & \text { if user } i \text { chooses } S \text { at state } s .\end{cases}$

The functions $C^{i}(s)$, describing the utility of player $i$ using the SC network at state $s$, are defined as follows

$C^{i}(1)=\mathbb{E}\left[c^{i}\left(h_{i}\right) \mid h_{i}>\Psi_{i}\right]=\frac{1}{\alpha_{i}} \int_{\Psi_{i}}^{\infty} c^{i}\left(h_{i}\right) \lambda_{i} e^{-\lambda_{i} h_{i}} d h_{i}$,

$C^{i}(0)=\mathbb{E}\left[c^{i}\left(h_{i}\right) \mid h_{i}<\Psi_{i}\right]=\frac{1}{1-\alpha_{i}} \int_{0}^{\Psi_{i}} c^{i}\left(h_{i}\right) \lambda_{i} e^{-\lambda_{i} h_{i}} d h_{i}$,

$c^{i}\left(h_{i}\right)$ above is the utility of player $i$ using $S$ when channel gain is $h_{i}$ and is defined as follows:

$$
c^{i}\left(h_{i}\right)=\log _{2}\left(1+\frac{p h_{i}}{\sigma^{2}}\right)
$$

On the other hand, the utilities $v_{k}^{i}$ of player $i$ using the MC in (4), when player $j$ applies policy $k$, are defined as follows

$$
v_{S S}^{i}=v_{\max }
$$

Next,

$$
\begin{aligned}
v_{M S}^{i} & =\left[\alpha_{j}+\left(1-\beta_{j}\right)\left(1-\alpha_{j}\right)\right] v_{\max } \\
& +\beta_{j}\left(1-\alpha_{j}\right) \min \left\{\frac{D G}{2}, v_{\max }\right\} \mathbb{I}_{\left\{\frac{D G}{2} \geq v_{\min }\right\}} \\
& +\beta_{j}\left(1-\alpha_{j}\right) \frac{v_{\max }}{2} \mathbb{\amalg}_{\left\{\frac{D G}{2}<v_{\min }\right\}}
\end{aligned}
$$

Finally,

$$
\begin{aligned}
v_{M M}^{i} & =\left(1-\beta_{j}\right) v_{\max }+\beta_{j} \min \left\{\frac{D G}{2}, v_{\max }\right\} \mathbb{1}_{\left\{\frac{D G}{2} \geq v_{\min }\right\}} \\
& +\beta_{j} \frac{v_{\max }}{2} \mathbb{I}_{\left\{\frac{D G}{2}<v_{\min }\right\}}
\end{aligned}
$$

The utilities for the last two cases reflect the fact that some arrivals may be blocked by the admission control when there is not enough resource for all the players - in that case we assume that some $k$ players (where $k$ is such that $\frac{D G}{k} \geq v_{\text {min }}$ ) willing to connect to the MC network are chosen at random (with equal probabilities) ${ }^{3}$ and they receive the service ${ }^{4}$. Note that the utilities defined above satisfy the following sets of inequalities:

$$
\begin{gathered}
C^{i}(1)>C^{i}(0), \\
v_{\max }=v_{S S}^{i} \geq v_{M S}^{i} \geq v_{M M}^{i}>\left(1-\beta_{j}\right) v_{\max } .
\end{gathered}
$$

\footnotetext{
${ }^{3}$ More precisely, the quantities appearing in (7) and (8) can be interpreted as follows: The term multiplied by $v_{\max }$ is the probability that $i$ is the only player using MC, the term appearing before $\min \left\{\frac{D G}{2}, v_{\max }\right\}$ and $\frac{v_{\max }}{2}$ is the probability that there are two players requesting connection to MC. Then if $\frac{D G}{2}<v_{\min }$, one player is chosen at random, so his utility is $v_{\max }$ with probability $\frac{1}{2}$ and 0 with probability $\frac{1}{2}$. Otherwise both players are admitted and their utility is $\min \left\{\frac{D G}{2}, v_{\max }\right\}$.

${ }^{4}$ In reality the ones chosen at random in our model would be those whose request was considered first.
} 


\section{B. The non-cooperative equilibrium}

Game theory has accentuated the importance of randomized games or mixed games. However, such a game does not find any significant role in most communication modems or source coding codecs since equilibria where each user randomly picks a decision at each time epoch cannot be used effectively there, as they amount to perpetual handover between networks.

Definition 1 (Bayes-Nash equilibrium). A strategy profile $\mathbf{P}_{\mathbf{i}}{ }^{B N E}, \forall i=1,2$ corresponds to a Bayes-Nash equilibrium (BNE) if, for all users, any unilateral switching to a different strategy cannot improve user's payoff at any state. Mathematically, this can be expressed by the following inequality, given the CDI about the other user $\forall \mathbf{Q}_{\mathbf{i}} \neq \mathbf{P}_{\mathbf{i}}^{B N E}$ and for $s_{i}=\{0,1\}$

$$
u_{i}\left(s_{i},\left(\mathbf{P}_{\mathbf{i}}^{B N E}, \mathbf{P}_{-\mathbf{i}}{ }^{B N E}\right)\right) \geq u_{i}\left(s_{i},\left(\mathbf{Q}_{\mathbf{i}}, \mathbf{P}_{-\mathbf{i}}{ }^{B N E}\right)\right) ;
$$

where the " $i$ " subscript on vector $\mathbf{P}$ stands for "except user $i$ ".

Proposition 1. The game considered in the paper always has a pure-strategy Bayes-Nash equilibrium. Moreover

(a) $(S S, S S)$ is an equilibrium iff $C^{i}(0) \geq v_{S S}^{i}$ for $i=1,2$.

(b) $(S S, M S)$ is an equilibrium iff $C^{1}(0) \geq v_{M S}^{1}$ and $C^{2}(1) \geq v_{S S}^{2} \geq C^{2}(0)$.

(c) $(S S, M M)$ is an equilibrium iff $C^{1}(0) \geq v_{M M}^{1}$ and $C^{2}(1) \leq v_{S S}^{2}$.

(d) $(M S, S S)$ is an equilibrium iff $C^{1}(1) \geq v_{S S}^{1} \geq C^{1}(0)$ and $C^{2}(0) \geq v_{M S}^{2}$.

(e) $(M S, M S)$ is an equilibrium iff $C^{i}(1) \geq v_{M S}^{i} \geq C^{i}(0)$ for $i=1,2$.

(f) $(M S, M M)$ is an equilibrium iff $C^{1}(1) \geq v_{M M}^{1} \geq$ $C^{1}(0)$ and $C^{2}(1) \leq v_{M S}^{2}$.

(g) $(M M, S S)$ is an equilibrium iff $C^{1}(1) \leq v_{S S}^{1}$ and $C^{2}(0) \geq v_{M M}^{2}$.

(h) $(M M, M S)$ is an equilibrium iff $C^{1}(1) \leq v_{M S}^{1}$ and $C^{2}(1) \geq v_{M M}^{2} \geq C^{2}(0)$.

(i) $(M M, M M)$ is an equilibrium iff $C^{i}(1) \leq v_{M M}^{i}$ for $i=1,2$.

Proof: The statements (a)-(i) are direct consequences of the definition of Bayes-Nash equilibrium and the form of payoff matrices. Next, using inequalities $(9,10)$, it is straightforward to show that we always have at least one of the conditions (a)-(i) satisfied.

The next proposition gives us some information on how the Nash-Bayes equilibria depend on the chosen values of the CQI thresholds $\Psi_{i}$.

Proposition 2. For any player $i$, if $\Psi_{i}$ is the small enough, player $i$ does not use policy $S S$ in equilibrium. If it is large enough, player $i$ does not use policy MM in equilibrium. Next define for $i=1,2$

$$
C^{i}(\infty)=\int_{0}^{\infty} c^{i}\left(h_{i}\right) \lambda_{i} e^{-\lambda_{i} h_{i}} d h_{i}
$$

If $C^{i}(\infty) \neq v_{\max }$ for $i=1,2$, then for all the values of the parameters of the model one of the three possibilities is true:

(a) For $\Psi_{1}$ and $\Psi_{2}$ small enough, both players use policy $M M$ in equilibrium,

(b) For $\Psi_{1}$ and $\Psi_{2}$ large enough, both players use policy $S S$ in equilibrium,

(c) For $\Psi_{i}$ small enough, $i$ uses policy $M M$ in equilibrium, while for $\Psi_{j}$ large enough $j$ uses policy $S S$ in equilibrium.

Proof: Note that ${ }^{5}$ :

1) when $\Psi_{i} \rightarrow 0, C^{i}(0)\left(\Psi_{i}\right) \rightarrow 0, C^{i}(1)\left(\Psi_{i}\right) \rightarrow C^{i}(\infty)$ and $v_{M S}^{j}\left(\Psi_{j}\right) \rightarrow v_{S S}^{j}$ for $i=1,2$;

2) when $\Psi_{i} \rightarrow \infty, C^{i}(0)\left(\Psi_{i}\right) \rightarrow C^{i}(\infty), C^{i}(1)\left(\Psi_{i}\right) \rightarrow$ $+\infty$ and $v_{M S}^{j}\left(\Psi_{j}\right) \rightarrow v_{M M}^{j}$.

Thus for $\Psi_{i}$ small enough, $C^{i}(0)\left(\Psi_{i}\right)<v_{M M}^{i}$, which by Proposition 1 implies that player $i$ does not use policy $S S$ in equilibrium. Analogously for $\Psi_{i}$ big enough, $C^{i}(1)\left(\Psi_{i}\right)>$ $v_{S S}^{i}$, and so player $i$ does not use $M M$ in equilibrium then.

Let us assume now that both $\Psi_{1}$ and $\Psi_{2}$ are either very small or very large and that in both situations the equilibrium is of type $(M S, M S)$. By Proposition 1, this implies that

$$
C^{i}(1)\left(\Psi_{i}\right) \geq v_{M S}^{i}\left(\Psi_{j}\right) \geq C^{i}(0)\left(\Psi_{i}\right)
$$

for $i=1,2$ in both situations ( $\Psi_{i} \mathrm{~s}$ small or large). Now taking into account properties 1) and 2) described above, we can pass to the limit as $\Psi_{i} \rightarrow 0, i=1,2$, getting:

$$
C^{1}(\infty) \geq v_{S S}^{i} \geq 0 \quad \text { for } i=1,2 .
$$

Analogously, passing to the limit as $\Psi_{i} \rightarrow \infty, i=1,2$, we obtain

$$
\infty \geq v_{M M}^{i} \geq C^{1}(\infty) \text { for } i=1,2 .
$$

But (12) together with (13) contradict (10) as long as $v_{\max } \neq C^{i}(\infty)$.

We prove similarly that neither of the situations enumerated below is possible: one of the profiles $(M S, M M)$ or $(M M, M S)$ is an equilibrium for $\Psi_{i} \mathrm{~s}$ approaching 0 and $(M S, M S)$ is an equilibrium for $\Psi_{i}$ s approaching infinity, or $(M S, M S)$ is an equilibrium for $\Psi_{i} \rightarrow 0$ and $(M S, S S)$ or $(S S, M S)$ is an equilibrium for $\Psi_{i} \rightarrow \infty$.

The result of this proposition can be interpreted in the following manner: for higher values of the CQI thresholds $\Psi_{i}$ the players prefer to use the SC rather than the MC and conversely, for low $\Psi_{i}$ s they prefer to use the MC. Interestingly, Proposition 2 also suggests that, rather than increasing the offered throughput $v$, the operator could control the equilibrium of its wireless users to maximize its own revenue by broadcasting appropriate CQI thresholds. This can lead the network to minimize its overall cost and users to a misleading association problem. Next, we

\footnotetext{
${ }^{5}$ In what is written below we use the convention that $C^{i}(s)\left(\Psi_{i}\right)$ means the value of $C^{i}(s)$ when $i$ 's CQI threshold is $\Psi_{i}$, similarly $v_{M S}^{i}\left(\Psi_{j}\right)$ is the value of $v_{M S}^{i}$ when his opponent's threshold is $\Psi_{j}$. Analogously for other functions.
} 
extend the analysis of the above mentioned problem using hierarchical approach.

\section{The hierarchical equilibrium}

In this section, we propose a methodology that transforms the above non-cooperative game into a Stackelberg game. Concretely, the MC network may guide users to an equilibrium that optimizes its own utility $U_{M C}$ if it chooses the adequate information to send. We first study the policy that maximizes the utility of the network, which is defined as the expected throughput of the MC network (we need to define it using two steps):

$$
\begin{aligned}
\mathcal{U}_{M C}\left(q_{1}, q_{2}\right) & =\left[q_{1}\left(1-q_{2}\right)+q_{2}\left(1-q_{1}\right)\right] v_{\max }+ \\
& q_{1} q_{2}\left[D G \mathbb{1}_{\left\{v_{\min } \leq \frac{D G}{2} \leq v_{\max }\right\}}+\right. \\
& \left.2 v_{\max } \mathbb{1}_{\left\{v_{\max }<\frac{D G}{2}\right\}}+v_{\max } \mathbb{I}_{\left\{\frac{D G}{2}<v_{\min }\right\}}\right],
\end{aligned}
$$

where $q_{1}$ and $q_{2}$ are the probabilities that player 1 , respectively player 2 , uses the MC. Consequently

$$
U_{M C}\left(\mathbf{P}, \Psi_{1}, \Psi_{2}\right)=\mathcal{U}_{M C}\left(q\left(\mathbf{P}_{1}, \Psi_{1}\right), q\left(\mathbf{P}_{2}, \Psi_{2}\right)\right)
$$

with $q\left(\mathbf{P}_{i}, \Psi_{i}\right)=\beta_{i}\left[\mathbb{I}_{\left\{\mathbf{P}_{i}=M M\right\}}+\left(1-e^{-\lambda_{i} \Psi_{i}}\right) \mathbb{\amalg}_{\left\{\mathbf{P}_{i}=M S\right\}}\right]$

Nevertheless, as it is not realistic to consider that the users will seek the global optimum, we show how to find the policy that corresponds to the Bayes-Stackelberg equilibrium where the $\mathrm{MC}$ tries to maximize $U_{M C}$ just by choosing the CQI thresholds, knowing that users will try to maximize their individual utility.

Definition 2 (Bayes-Stackelberg equilibrium). By denoting $\left(\Psi_{1}^{B S E}, \Psi_{2}{ }^{B S E}\right)$ the strategy profile of the $M C$ at a Bayes-Stackelberg equilibrium (BSE), this definition translates mathematically as

$\left(\Psi_{1}^{B S E}, \Psi_{2}^{B S E}\right) \in \arg \max _{\mathbf{\Psi}_{1}, \mathbf{\Psi}_{2}} U_{M C}\left(\mathbf{P}^{B N E}\left(\Psi_{1}, \Psi_{2}\right), \Psi_{1}, \Psi_{2}\right)$,

where $\mathbf{P}^{B N E}\left(\Psi_{1}, \Psi_{2}\right)$ is any Bayes-Nash equilibrium in the game of the previous section with CQI thresholds equal to $\Psi_{1}, \Psi_{2}$.

We next exemplify our general analysis by investigating the possibility of considering three scenarios for the choice of $\Psi_{1}$ and $\Psi_{2}$ :

1) Centralized model - the MC chooses both $\Psi_{i} \mathrm{~s}$ and the policies for the players, aiming to maximize $U_{M C}$. Formally, the centralized strategy is the one satisfying

$$
\left(\Psi_{1}{ }^{C}, \Psi_{2}{ }^{C}, \mathbf{P}^{C}\right) \in \arg \max _{\Psi_{1}, \Psi_{2}, \mathbf{P}} U_{M C}\left(\mathbf{P}, \Psi_{1}, \Psi_{2}\right),
$$

2) Stackelberg model - there are two stages: at the first one the MC chooses both $\Psi_{i}$ s given the information about the distributions of $\left(h_{i}, b_{i}\right)$ aiming to maximize the throughput of the MC network at the second stage, when players play the game from the last section. The proposed approach can be seen as intermediate scheme between the centralized model and the fully non-cooperative model,
3) Fully non-cooperative model - the game has two stages: at the first one, players choose their $\Psi_{i}$ s given the information they have about the distributions of $\left(h_{i}, b_{i}\right)$ aiming to maximize their expected throughput at the second stage; at the second stage they choose a policy depending on actual $\left(s_{i}, b_{i}\right)$ as in the model of the last section. Formally, a fully non-cooperative strategy is any one satisfying

$\Psi_{i}^{N C} \in \arg \max _{\Psi_{i}} \mathbb{E}\left[u_{i}\left(s_{i}, \mathbf{P}^{B N E}\left(\Psi_{i}, \Psi_{j}^{N C}\right)\right)\right] ;$ for $i=1,2$

with $\mathbf{P}^{B N E}\left(\Psi_{i}, \Psi_{j}^{N C}\right)$ being any Bayes-Nash equilibrium in the game of the previous section.

Remark 2. The name fully non-cooperative suggests a different model, without two stages, where the decision both about the CQI thresholds and about players' policies is made by the users at the same time. As it will be shown in Proposition 4, the two models are equivalent. The two-step procedure that we propose only makes a clear distinction between stage one when no communication is needed, because all the data necessary to compute the $\Psi_{i}$ is available to all the players and the MC, and stage two when the information about channel quality has to be reported to the players to assign them to MC or to SC. Thus, as in all the other scenarios that we provide, the amount of information that has to be transmitted during the game is very limited.

Below, we analyze the behavior of the MC and the players at the equilibria of each of these models.

\section{Proposition 3.}

1) In the centralized model, the MC chooses any $\Psi_{1}$ and $\Psi_{2}$, and $M M$ policy for both users.

2) In the Stackelberg model, the MC does the following steps:

a) Computes $C^{i}(\infty)$ for $i=1,2$. If $C^{1}(\infty) \leq v_{M M}^{1}$ and $C^{2}(\infty) \leq v_{M M}^{2}$, then it chooses any $\Psi_{1}<\Psi_{1}^{* *}$ and $\Psi_{2}<\Psi_{2}^{* *}$ with $\Psi_{i}^{* *}$ satisfying $C^{1}(1)\left(\Psi_{1}^{* *}\right)=v_{M M}^{1}$ and $C^{2}(1)\left(\Psi_{2}^{* *}\right)=v_{M M}^{2}$ and then users both play $M M$ in the second stage.

b) It finds ${ }^{6} \Psi_{1}, \Psi_{2}$ solving three maximization problems:

b1: maximize $\mathcal{U}_{M C}\left(\beta_{1}\left(1-e^{-\lambda_{1} \Psi_{1}}\right), \beta_{2}\right)$ subject to $v_{M S}^{1}\left(\Psi_{2}\right) \geq C^{1}(\infty), C^{2}(1)\left(\Psi_{2}\right) \geq v_{M M}^{2} \geq$ $C^{2}(0)\left(\Psi_{2}\right)$;

b2: maximize $\mathcal{U}_{M C}\left(\beta_{1}, \beta_{2}\left(1-e^{-\lambda_{2} \Psi_{2}}\right)\right)$ subject to $C^{1}(1)\left(\Psi_{1}\right) \geq v_{M M}^{1} \geq C^{1}(0)\left(\Psi_{1}\right)$, $v_{M S}^{2}\left(\Psi_{1}\right) \geq C^{2}(\infty)$;

b3: maximize $\mathcal{U}_{M C}\left(\beta_{1}\left(1-e^{-\lambda_{1} \Psi_{1}}\right), \beta_{2}\left(1-e^{-\lambda_{2} \Psi_{2}}\right)\right)$ subject to $C^{1}(1)\left(\Psi_{1}\right) \geq v_{M S}^{1}\left(\Psi_{2}\right) \geq C^{1}(0)\left(\Psi_{1}\right)$, $C^{2}(1)\left(\Psi_{2}\right) \geq v_{M S}^{2}\left(\Psi_{1}\right) \geq C^{2}(0)\left(\Psi_{2}\right)$.

It chooses the biggest of the three value functions $V_{b}$ (if some of these problems has no solutions we assume the value of its value function is 0 ) and $\Psi_{1}$, $\Psi_{2}$ solving this problem is what it plays at the first

${ }^{6}$ If some problem does not depend on $\Psi_{i}$, we take $\Psi_{i}=0$. 
stage of the game. At the second stage players choose policies $(M S, M M),(M M, M S)$ and $(M S, M S)$ if the solution of problem $b 1, b 2$ and $b 3$ (respectively) was chosen by the MC.

3) In the fully non-cooperative model, the players in equilibrium choose $\Psi_{1}=\Psi_{1}^{*}$ and $\Psi_{2}=\Psi_{2}^{*}$ satisfying

$$
c^{1}\left(\Psi_{1}^{*}\right)=v_{M S}^{1}, \quad c^{2}\left(\Psi_{2}^{*}\right)=v_{M S}^{2}
$$

and then both use a MS policy.

For the clarity of the exposition, proofs are given in the Appendix. What we see in this proposition is that when the MC can decide on the behavior of the users, it forces them to use the MC. In other cases (when users can decide on their behavior, but are given only partial information), the users' interest is to choose the CQI thresholds somewhere in the middle of the channel gain range. On the other hand, the MC has an incentive to choose CQI thresholds either very low (first case in the Stackelberg scenario) or very high (the second case). Both these choices give little information for the user about actual channel condition, which is what he wants to avoid. It is interesting and somewhat surprising that the optimal policy of the MC in the Stackelberg game can be both giving high or low values of CQI thresholds. This can however be explained when we understand the meaning of these two situations - very low value of the threshold means that no information about the channel state is given. In this case, when both users connect to the MC, this corresponds to the choice of the MC. Now, if in the "no information" case players choose the SC network, then the MC tries to divide the range of $h_{i}$ into a small (in terms of probability) part when the players use the SC and, a large one when they use the MC. This is done by giving the highest possible CQI threshold below which the players would have an incentive to use rather the MC than the SC. This explains why the $\mathrm{MC}$ has an incentive to choose CQI thresholds very high in this case.

In the next proposition, we show that the strategies chosen by the players in the fully non-cooperative scenario of Proposition 3 are also equilibria in two other noncooperative models, differing from the one considered above by the time the decisions are made by the players or the amount of information available to them.

Proposition 4. Consider two alternative non-cooperative models:

(M1) The game has one stage, at which the players choose both CQI thresholds and policies they apply, aiming to maximize their own throughput. They base their choices on the information about distributions of $\left(h_{i}, b_{i}\right)$.

(M2) The game has one stage, at which the players choose whether they want to connect to MC or SC, aiming to maximize their own throughput. Each player knows the distribution of $\left(h_{j}, b_{j}\right)$ of the opponent and exact values of his own $h_{i}$ and $b_{i}$.
If $\Psi_{1}^{*}$ and $\Psi_{2}^{*}$ are CQI thresholds chosen in part 3) of Proposition 3, then $\Psi_{1}^{*}$ - and $\Psi_{2}^{*}$-threshold policies (that is - MS policies with $\Psi_{i}^{*}$ CQI thresholds) are also in equilibrium in models (M1) and (M2).

The final result of this section is given without proof, which is straightforward. In that result, we give the method to compute the PoA for our model. Remind that the PoA measures how good the system performance is when users play selfishly and reach the NE instead of playing to achieve the social optimum obtained in the centralized model with utility $\mathcal{U}_{M C}\left(\beta_{1}, \beta_{2}\right)$. Thus the PoA when players use strategy profile $\mathbf{P}$ is

$$
\operatorname{Po} A=\frac{\mathcal{U}_{M C}\left(\beta_{1}, \beta_{2}\right)}{\mathcal{U}_{M C}\left(q\left(\mathbf{P}_{1}, \Psi_{1}\right), q\left(\mathbf{P}_{2}, \Psi_{2}\right)\right)} .
$$

Thus, Proposition 3 implies that

Corollary 1. The PoA in the Stackelberg model equals 1 whenever $C^{i}(\infty) \leq v_{M M}^{i}$ for $i=1,2$. When for some $i$, $C_{M M}^{i}(\infty)>v$, then the PoA is equal to

$$
\frac{\mathcal{U}_{M C}\left(\beta_{1}, \beta_{2}\right)}{V_{b}}
$$

where $V_{b}$ is the value of the objective function computed in Proposition 3.

In fully non-cooperative model,

$$
\operatorname{PoA}=\frac{\mathcal{U}_{M C}\left(\beta_{1}, \beta_{2}\right)}{\mathcal{U}_{M C}\left(\beta_{1}\left(1-e^{-\lambda_{1} \Psi_{1}^{*}}\right), \beta_{2}\left(1-e^{-\lambda_{2} \Psi_{2}^{*}}\right)\right)},
$$

where $\Psi_{1}^{*}$ and $\Psi_{2}^{*}$ satisfy (15).

The above corollary is just a rewriting of the Proposition 3 using different language.

\section{THE MULTI-USER CASE}

Now, let us consider the case where instead of two we have $n$ users choosing to connect either to the SC or to the MC network. Again we assume that the information about the channel quality that user $i$ possesses is limited to that about the distributions of states $\left(s_{j}, b_{i}\right)$ of each of the players (including $i$ ), that is about $\alpha_{j}$ (or $\lambda_{j}$ ) and $\beta_{j}$ and to exact information about his own current state $\left(s_{i}, b_{i}\right)$ (but not about exact value of $h_{i}$ ). We make two additional assumptions about the model considered in this section. The first one is that the model is symmetric, that is all the values $\beta_{i}, \lambda_{i}$ and $\Psi_{i}$ defining it, are the same for each of the players (and equal to $\beta, \lambda$ and $\Psi$ respectively). The second one is that $v_{\max } \geq 2 v_{\min }$. Both of them aim at simplifying the notation used in our considerations. On the other hand, we believe that some counterparts of all our results are true also without these assumptions, so they can be made without serious limitation of generality.

\section{A. Utilities}

Again we assume that each of the players uses one of the three policies $S S, M S, M M$, where first letter stands for a player's action when his channel is bad, and the second 
one when his channel is good. As it is troublesome to write down the policies for each of $n$ players, we will make use of the fact that the game is symmetric, writing instead of the policy profile a policy statistics $\mathbf{K}=\left[k_{M M}, k_{M S}\right]$ with $k_{M M}$ denoting the number of players applying policy $M M$ and $k_{M S}-$ of players applying $M S$. Of course the number of those using policy $S S$ is $n-k_{M M}-k_{M S}$, so we will omit it. Given $\mathbf{K}$, we can define user $i$ 's utility in state $s=0,1$ as $^{7}$

$$
u_{i}(s, \mathbf{K})= \begin{cases}v_{\mathbf{K}_{-i} ;} & \text { if user } i \text { chooses } M \text { at state } s \\ C(s) ; & \text { if user } i \text { chooses } S \text { at state } s\end{cases}
$$

where $C(s)$ is defined as $C^{i}(s)$ for two-user case, with the superscript omitted because the model is symmetric, while the values $v_{\mathbf{K}_{-i}}$, describing the utility of player $i$ using the MC when his opponents use policies described by $\mathbf{K}$, are similarly as for the two-user case ${ }^{8}$ :

$$
\begin{aligned}
v_{[k, l]} & =\sum_{r=0}^{k} \sum_{q=0}^{l} \sum_{w=0}^{q} \beta^{r+q}(1-\beta)^{k+l-r-q}\left(\begin{array}{c}
k \\
r
\end{array}\right)\left(\begin{array}{c}
l \\
q
\end{array}\right)\left(\begin{array}{c}
q \\
w
\end{array}\right) \\
& \cdot e^{-\lambda(q-w) \Psi}\left(1-e^{-\lambda \Psi}\right)^{w} \frac{\min \left\{k^{*}, r+w+1\right\}}{r+w+1} \\
& \cdot \min \left\{\frac{D G}{\min \left\{k^{*}, r+w+1\right\}}, v_{\max }\right\}
\end{aligned}
$$

where $k^{*}=\max \left\{k: \frac{D G}{k} \geq v_{\min }\right\}$ is the biggest number of users that can be admitted to MC without decreasing their throughputs below $v_{\min }$ (remember that, as in the twoplayer case, the utility defined above is the expected value of user's throughput, already taking into account the admission control).

\section{B. The equilibria}

Below, we give a generalization of Proposition 1 for the $n$-user case.

Proposition 5. The symmetric $n$-user game considered in the paper always has a pure-strategy Bayes-Nash equilibrium of one of seven types:

(a) When $v_{[k-1, l]} \geq C(1) \geq v_{[k, l-1]} \geq C(0) \geq v_{[k, l]}$, then any profile where $k$ players use policy $M M, l$ players use policy $M S$, and all the others play $S S$ is an equilibrium.

(b) When $v_{[k-1,0]} \geq C(1) \geq C(0) \geq v_{[k, 0]}$ then any profile where $k$ players apply policy $M M$ and the remaining $n-k$ players use policy $S S$ is an equilibrium.

(c) When $v_{[k-1, n-k]} \geq C(1) \geq v_{[k, n-k-1]} \geq C(0)$ then any profile where $k$ players apply policy $M M$ and the remaining $n-k$ players use policy $M S$ is an equilibrium.

(d) When $v_{[n-1,0]} \geq C(1)$ then the profile where all the players use policy $M M$ is an equilibrium.

(e) When $C(1) \geq v_{[0, k-1]} \geq C(0) \geq v_{[0, k]}$, then any profile where $k$ players use policy $M S$ and all the others play $S S$ is an equilibrium.

\footnotetext{
${ }^{7}$ Notation $\mathbf{K}_{-\mathbf{i}}$ used below denotes policy statistics defined as in the two-user case but without policy of user $i$.

${ }^{8} \mathrm{Of} \mathrm{S}$ as this is a generalization of the formulas for $v_{k}^{i}$ given in Section III and it applies for any $n \geq 2$, in particular $v_{M M}^{i} \equiv v_{[1,0]}, v_{S C}^{i} \equiv$ $v_{[0,1]}$ and $v_{S S}^{i} \equiv v_{[0,0]}$ when $n=2$ and players are symmetric.
}

(f) When $C(1) \geq v_{[0, n-1]} \geq C(0)$ then the profile where all the players use policy $M S$ is an equilibrium.

(g) When $C(0) \geq v_{[0,0]}$ then the profile where all the players use policy $S S$ is an equilibrium.

We give a corollary to this proposition. It gives a kind of consistency property for equilibria in games for different values of $n$.

\section{Corollary 2.}

(a) Suppose that a profile where at least one player uses policy $S S$ and the number of players using policies $M M$ and $M S$ is $k$, is an equilibrium in n-user symmetric game. Then it is also an equilibrium in any $m$ user game defined with the same parameters $\beta, \lambda$ and $\Psi$ and $m \geq k$.

(b) Moreover for any fixed parameters $\beta, \lambda$ and $\Psi$ there exists an $m$ such that for any $n>m$ at least $n-$ $m$ players use policy $S S$ in any equilibrium in $n$-user game.

Proof: Note that $v_{[k, l]}$ does not depend on the number of players in the game $n$, only on the number of those who use one of the policies $M S$ or $M M$. Just this implies part (a). Part (b) is due to the fact that $v_{[k, l]} \rightarrow 0$ as either $k \rightarrow \infty$ or $l \rightarrow \infty$.

The next proposition generalizes the results for hierarchical model included in Proposition 3 for $n$-user symmetric games. We consider all the above scenarios, yet in scenario 3) we only show that the equilibrium is symmetric, but assume that players may act asymmetrically in general. To do so we look for symmetric equilibria in the model (which exist, but we believe are not the only ones possible there, even though the model itself is symmetric). The rationale behind this simplification is twofold: firstly - considering asymmetric equilibria would cause various problems with notation; secondly and most important - we believe that asymmetric equilibria, where users may have different functional form of their strategies, are harder to justify, as they would require prior coordination among the devices to agree on which equilibrium is played.

The MC utility, defined as before, as the expected throughput of the MC network, can be now written as:

$$
\begin{aligned}
U_{M C}([k, l], \Psi) & =\sum_{r=0}^{k} \sum_{q=0}^{l} \sum_{w=0}^{q} \beta^{r+q}(1-\beta)^{k+l-r-q} \\
& \cdot\left(\begin{array}{c}
k \\
r
\end{array}\right)\left(\begin{array}{l}
l \\
q
\end{array}\right)\left(\begin{array}{c}
q \\
w
\end{array}\right) e^{-\lambda(q-w) \Psi}\left(1-e^{-\lambda \Psi}\right)^{w} \\
& \cdot \min \left\{D G, v_{\max }(w+r)\right\} .
\end{aligned}
$$

\section{Proposition 6.}

1) In the centralized model, the MC chooses any value of $\Psi$ and $M M$ policy for all the users.

2) In the Stackelberg model, the MC computes $C(\infty)$ (see (11)) and finds $k^{* *}$ such that

$$
v_{\left[k^{* *}-1,0\right]}>C(\infty) \geq v_{\left[k^{* *}, 0\right]} .
$$


If such a $k^{* *}$ does not exist, it sets $k^{* *}=0$. Next:

(a) If $n \leq k^{* *}$ then at the equilibrium the MC chooses any $\Psi$ such that $v_{[n-1,0]} \geq C(1)(\Psi)$, and all the players use policy $M M$.

(b) If $n>k^{* *}$ then:

b1) for any $k$ such that $k^{* *} \leq k \leq n$ and any $0 \leq l \leq k^{* *}$ the MC does the following steps: it finds $\underline{\Psi}$ such that

$$
C(1)(\underline{\Psi})=v_{[l-1, k-l]}(\underline{\Psi}) .
$$

If such a $\underline{\Psi}$ does not exist or $C(0)(\underline{\Psi})<$ $v_{[l, k-l]}(\underline{\Psi})$, it puts $P(k, l)=0$. Otherwise it finds $\bar{\Psi}$ such that

$$
C(0)(\bar{\Psi})=v_{[l, k-l-1]}(\bar{\Psi})
$$

If such a $\bar{\Psi}$ does not exist, it puts $\bar{\Psi}=\infty$. Finally, if $P(k, l)$ has not been defined yet, it takes $\Psi(k, l)=\min \{\bar{\Psi}, \underline{\Psi}\}$ and computes

$$
P(k, l)=U_{M C}([l, k-l], \Psi(k, l)) .
$$

b2) it chooses $k_{\max }$ and $l_{\max }$ with the biggest value of $P(k, l)$ (which equals the MC utility at equilibrium). The choice of $\Psi\left(k_{\max }, l_{\max }\right)$ at the first stage and any profile of policies where $l_{\max }$ players use policy $M M$ and $k_{\max }-l_{\max }$ play $M S$ will then be an equilibrium.

3) In the fully non-cooperative model, the players choose $\Psi^{*}$ satisfying

$$
c\left(\Psi^{*}\right)=v_{[0, n-1]}\left(\Psi^{*}\right),
$$

and then all use MS policy at the second stage of the game.

In order to compare the network utility under different modes, we give two corollaries to this proposition.

Corollary 3. The PoA in the n-user hierarchical model can be computed as

$$
\operatorname{PoA}=\frac{U_{M C}([n, 0], \Psi)}{U_{M C}(\mathbf{K}, \Psi)}
$$

(where $U_{M C}([n, 0], \Psi)$ is the maximum value of the MC's utility obtained in scenario 1) of Proposition 6), which is independent of $\Psi$. Moreover:

1) In the Stackelberg model it is either equal to 1 when $k \leq k^{* *}$, or satisfies

$$
P o A=\min _{k^{* *} \leq k \leq n, 0 \leq l \leq k} \frac{U_{M C}([n, 0], \Psi)}{P(k, l)}
$$

with $k^{* *}$ and $P(k, l)$ defined as in Proposition 6.

2) In the fully non-cooperative model it can be computed as:

$$
\operatorname{PoA}=\frac{U_{M C}([n, 0], \Psi)}{U_{M C}\left([0, n], \Psi^{*}\right)}
$$

with $\Psi^{*}$ defined as in Proposition 6.
The corollary is again just a rewriting of the results from Proposition 6 with the stress made on network utilities rather than strategies of the players. It shows that exactly the same procedure, used to find the equilibrium policies, can be applied to evaluate the performance of the network.

\section{The Non-COOPERATIVE Full CSI Model}

In this section, we consider a model where every player has full information about the quality of channels and amount of data to be transmitted by each of the players. It means that the players use exact information about their own CSI in their decisions, not only the indication whether it is good or bad, and also that they use exact information about that of the others, not limited to their distributions. Notice that the non-cooperative full CSI scheme is not realistic as it is not feasible to consider that the network operator will divulge users' CSI to other users. This will thus serve as as a reference model in our numerical analyses in order to demonstrate how much gain may be exploited through considering such a non-cooperative full CSI solution with respect to the other schemes we considered which uses partial CDI.

Suppose $^{9}$ that the number of transmitting players is $n$. Further, we assume that each of the players knows exact values of all the channel power gains $h_{j}$. This means that strategies $\mathbf{P}_{i}$ in this game will map any power gain profile $\left(h_{1}, \ldots, h_{n}\right)$ into the set $\{M, S\}$.

\section{A. Utilities}

Consequently, the utility of a player will depend on his strategy $\mathbf{P}_{i}$ and on number $\mathbf{k}_{-i}$ of those except him choosing $M$ in the following way:

$$
\widehat{u}_{i}\left(\mathbf{P}_{i}, \mathbf{k}_{-i}\right)= \begin{cases}v_{\mathbf{k}_{-i}} ; & \text { if user } i \text { chooses } M, \\ c\left(h_{i}\right) ; & \text { if user } i \text { chooses } S\end{cases}
$$

with $c\left(h_{i}\right)$ defined as $c^{i}\left(h_{i}\right)$ for two-user case, with the superscript omitted because the model is symmetric, and the values $v_{\mathbf{k}_{-i}}$, describing the utility of player $i$ using $\mathbf{M}$ when the number of others using $\mathbf{M}$ is $\mathbf{k}_{-i}$ defined as

$$
v_{k}=\frac{\min \left\{k^{*}, k+1\right\}}{k+1} \min \left\{\frac{D G}{\min \left\{k^{*}, k+1\right\}}, v_{\max }\right\}
$$

again with $k^{*}=\max \left\{k: \frac{D G}{k} \geq v_{\text {min }}\right\}$.

\section{B. Equilibria}

Below, we show how to find equilibria in the noncooperative full CSI model described above. To formulate our main result, we will need some additional notation. Let $\pi(i)=\left|\left\{j \leq n: h_{j} \leq h_{i}\right\}\right|$, that is, $\pi(i)=l$ means that $h_{i}$ is the $l$-th smallest channel gain. The following result will hold for our $n$-player non-cooperative full CSI game.

\footnotetext{
${ }^{9}$ The use of $n$ is a little abuse of notation, because here we do not assume that users transmit only with a given probability, like in all the other schemes we considered.
} 
Proposition 7. The n-user non-cooperative full CSI game considered in the paper always has a pure-strategy Nash equilibrium. Moreover, any ${ }^{10}$ equilibrium can be identified in the following way: if $\widehat{k}$ is such that

$$
c\left(h_{i}\right) \leq v_{\widehat{k}} \leq c\left(h_{j}\right)
$$

for $i$ and $j$ such that $\pi(i)=\widehat{k}$ and $\pi(j)=\widehat{k}+1$ (with the following exceptions: when $v_{n}>c\left(h_{i}\right)$ for any $i$, then we take $\widehat{k}=n$, while when $v_{1}<c\left(h_{i}\right)$ for any $i$, then we take $\widehat{k}=0)$, then each player $j$ such that $\pi(j) \leq \widehat{k}$ plays $M$ in equilibrium, while each player $j$ such that $\pi(j)>\widehat{k}$ plays $S$ in the equilibrium.

\section{IMPLEMENTATION ISSUES}

One of the major goals of the proposed approach is to delegate the access selection decisions to the devices, while providing the network with more control over SC access selections. The main idea behind this is that the device is in the unique position to make the best final determination of when traffic can be transported over SC (e.g., based on real-time radio conditions, type of pending traffic, device conditions such as mobility and battery status, etc.). This led us to propose a network-assisted approach where both the device and the network are involved in choosing the best network selection decisions. From an implementation point of view, the proposed hierarchical scheme requires little communication between players. The steps of the proposed approach (described in Fig. 2) are as follows: The SC collects the channel gain estimates $h_{i}$ received from UEs within its coverage. Then, with the goal of reducing the overhead backhaul traffic, the MC sends to the SC the CQI threshold $\Psi$ computed at the previous scheduling stage, and the SC, in turn, sends back a one-bit indication of the quality of the channel (either in bad or good state) to the MC. Next, the MC distributes the updated value of $\Psi$ computed using algorithm from Prop. 5 or Prop. 6, along wih the estimate(s) of $\alpha$ computed using Eq. (3). Based on that, UEs select their equilibrium strategies, compute their expected utilities and return their network preference lists to the MC. Finally, the MC decides to which cell it better to attach each UE (either $\mathrm{MC}$ or SC), and each network computes its utility.

Notice that the algorithm for computing the $\Psi$ is run at the macro BS. However, this is not a restriction of the proposed approach, as it can also be run at the user side and not transmitted. Obviously, these are not trivial computations, it is thus better to run them once at the macro BS and send the $\Psi$ to all the users.

The next proposition points out an important feature of the algorithm for finding the solution to the Stackelberg game.

Proposition 8. The complexity of the algorithm for finding the equilibrium of the Stackelberg model, described in part 2) of Proposition 6 is $\mathcal{O}\left(n^{4}\right)$ (where $n$ is the number of users).

${ }^{10}$ If each channel gain is different, then the equilibrium will be unique.

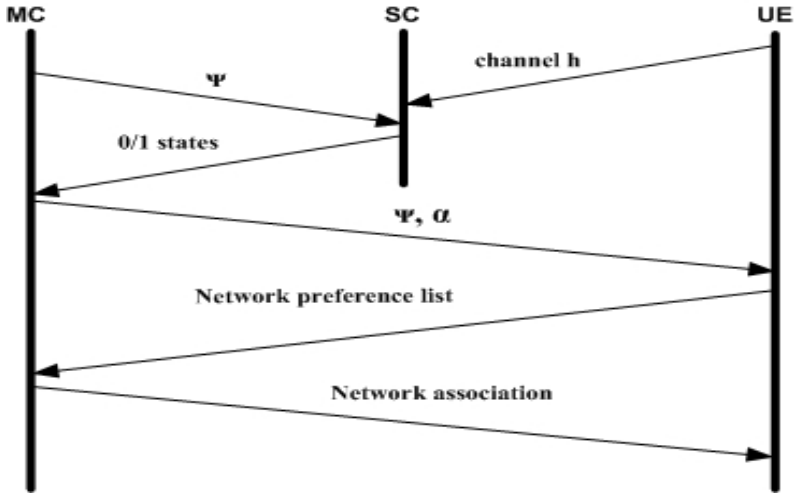

Fig. 2. Schematic diagram showing the set of parameters/values communicated among $\mathrm{MC} / \mathrm{SC}$ and $\mathrm{UE}$, and the set of steps implemented at $\mathrm{MC} / \mathrm{SC}$ and UE.

\section{NUMERICAL RESUlTS}

We consider a scenario of an operator providing subscribers with a streaming service available through a large $\mathrm{MC}$ coexisting with a SC. users require a minimal throughput of $350 \mathrm{Kbps}$ and can profit from throughputs up to 2.8 Mbps in order to enhance video quality (i.e., $v_{\min }=0.35$ Mbps and $v_{\max }=2.8 \mathrm{Mbps}$ ). As mentioned before, users are characterized by the distribution of their uplink channel and the distribution of their demand. In order to validate our theoretical findings, we obtain users' actions at the equilibrium defined by users' decisions to connect to the SC or the MC at low and high channel state. In particular, we present extensive results for the hierarchical (Stackelberg) equilibrium, non-cooperative (Nash) equilibrium and compare them with the centralized (optimal) strategy. To do so, we define a set of $n \in\{2, \ldots, 50\}$ competing users with Rayleigh fading characterized by parameter $\lambda$, assumed to be symmetric for the multi-user case. For the following set of simulations, we take $\lambda=8 \mathrm{~dB}$ with a line of sight channel gain of $30 \mathrm{~dB}$. Each user thus experiences an average channel gain at $26 \%$ of the maximum transmission channel gain. We also set the demand load $\beta=0.5$ for every user, and the channel state $\alpha$ derives from $\lambda$ and $\Psi$ as in Eq. (3). 1000 scenarios are simulated to remove the random effects from Rayleigh fading. To show the influence of user's CQI threshold $\Psi$ on the different equilibrium strategies, we compute the users' best responses for different values of the threshold $\Psi$. It is then possible to compute the non-cooperative Bayes-Nash equilibrium strategies and the related users' utilities obtained at the equilibrium. For the hierarchical Stackelberg equilibrium, given the action of the MC, i.e., the CQI threshold $\Psi$, we compute the bestresponse function of the users defined as the action that maximizes users' utilities given the action of the MC. The network utility is defined as the average throughput obtained by a user selecting the MC. Finally, under the formerly defined policy statistics $\mathbf{K}=\{k, l\}$, the ratio number of users connected to system $a$ (with $a=M$ for the MC and 
$a=S$ for the SC), $\mathcal{L}(a)$, can be expressed as follows:

$$
\begin{gathered}
\mathcal{L}(M)=(k+l \alpha) / n \\
\mathcal{L}(S)=(n-k-l \alpha) / n
\end{gathered}
$$

\section{A. Performances as a function of the CQI threshold $\Psi$}

In Figures 3 and 4, we plot the ratio number of users connected per system according to the strategies at the equilibrium as a function of the normalized CQI threshold $\Psi$. First, as claimed by Prop. 61 ), we find that, for the centralized policy, all users choose policy $M M$ for any value of $\Psi$ fixed by the MC. Second, in accordance to the result of Prop. 6, the ratio numbers of users connected to the $\mathrm{MC}$ and the SC networks match the announced equilibrium policies for some specific values of the CQI threshold. In particular, we find that, as we increase the value of $\Psi$, users have more incentive to choose strategy $S$ at the equilibrium. Asymptotically, when $\Psi$ grows large, users avoid choosing strategy $M$ at equilibrium, since the channel will be claimed to be always bad. Next, for the values of $\Psi \leq 0.5$ when $n=8$ and $\Psi \leq 0.45$ when $n=40$, all the players select $M$ below $\Psi$. Above these values some players start using $S S$ policy. Similarly, for $\Psi \geq 0.33$ when $n=8$ and $\Psi \geq 0.3$ when $n=40$, all the players select $S$ above $\Psi$, while below these values some select $M M$ policy.

Further, in the case of $n<k^{* *}$ in Figure 3, we observe that for $\Psi \leq 0.05$, all the players use $M$. As a consequence, the value of $\Psi=0.05$ is chosen in hierarchical equilibrium, as it guarantees the maximal use of the MC network. On the other hand, the CQI threshold at the non-cooperative equilibrium is reached for $\Psi^{*}=0.5$, where only $20 \%$ of the players use MC network. It clearly suggests a better efficiency of the Stackelberg formulation in this case.

In the case of $n>k^{* *}$ presented in Figure 4, the percentage of users associated with the MC network further decreases to $16 \%$ for $\Psi^{*}=0.3$. In the Stackelberg case, the $\Psi$ increases to 0.45 , with $28 \%$ of the players choosing $M$. In both cases, all the players use $M S$ policy, but higher $\Psi$ implies that the use of strategy $M$ is established more often. Lack of players using $M M$ policy in the Stackelberg equilibrium is in accordance with Prop. 6 2)-(b), and rather intuitive, as the MC becomes saturated when too many players want to select it.

\section{B. Performances as a function of the number of users $n$.}

1) CQI threshold: In Figure 5, we compare the values of the CQI thresholds at the non-cooperative equilibrium $\Psi^{*}$ and at the Stackelberg equilibrium $\Psi\left(k_{\max }, l_{\max }\right)$ for increasing number of players. We observe that, $\Psi\left(k_{\max }, l_{\max }\right)$ is for $n \leq k^{* *}$ very small, as this guarantees the connection of all the users to the MC network both above and below the CQI threshold, while for $n>k^{* *}$ it rapidly increases to minimize the probability of connecting to the SC network when users start applying $M S$ policy at the equilibrium. Further, we see that $\Psi$ at the Stackelberg equilibrium decreases with the number of entering users. This is because the individual throughput of the users

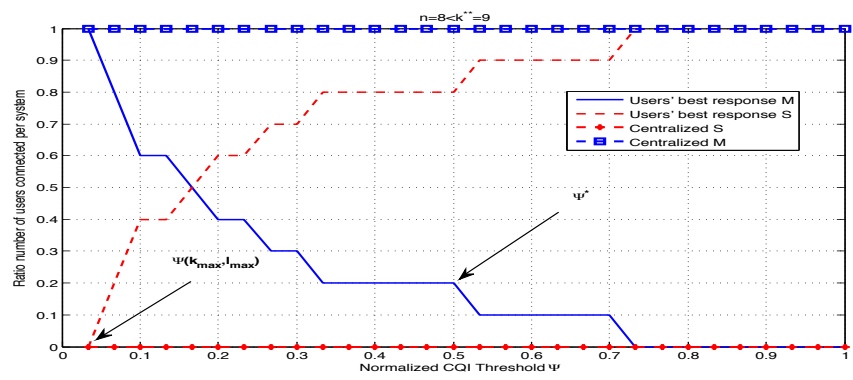

Fig. 3. Ratio number of users connected per system at the equilibrium as a function of $\Psi$ for $n=8$.

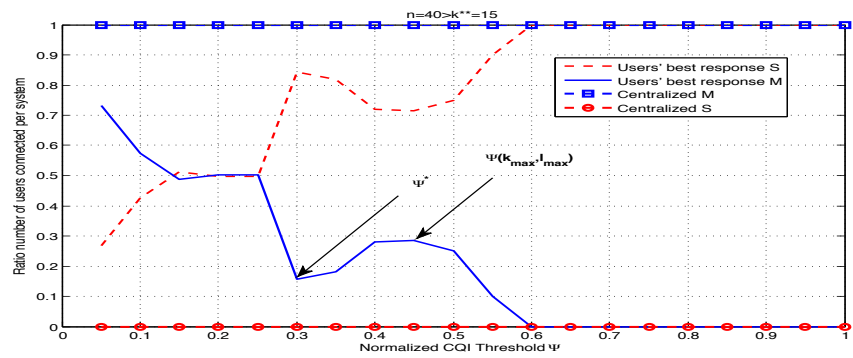

Fig. 4. Ratio number of users connected per system at the equilibrium as a function of $\Psi$ for $n=40$.

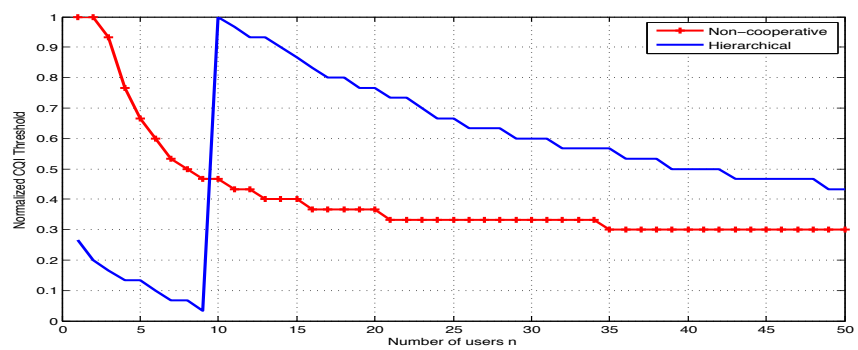

Fig. 5. CQI threshold at the equilibrium as function of increasing number of competing users $n$.

connected to the MC decreases as $n$ grows (see Eq. (1)), while there is no such effect for the SC network (see Eq. (2)). Thus users should be more attracted by the SC network, which results in a smaller $\Psi\left(k_{\max }, l_{\max }\right)$ above which users connect to the SC network. Even when $v_{\min }$ is achieved, the throughput for a user connected to the MC decreases, because we measure it as the expected value, and this encompasses the situation when a user prefers to connect to the MC even though he may end up not connected to it because the MC is already saturated. Nevertheless, the probability that the user chooses to connect to the MC remain big enough for him to make it more attractive than the SC network when $h_{i}$ is small. The CQI threshold at the noncooperative equilibrium $\Psi^{*}$ follows the same decreasing trend, but stays significantly below $\Psi\left(k_{\max }, l_{\max }\right)$. It can be noted though, that they become closer as $n$ increases.

2) Users' utilities: Figure 6 depicts users' utilities for increasing number of users. As expected, we first observe that the hierarchical and the non-cooperative models give the same outcome. In fact, the user's utility is calculated upon the estimates of the channel state of the user itself and other users based on the MC indications of $\Psi$ at the equilibrium. In the hierarchical case, the equilibrium may not exist, in which case any value of $\Psi$ can be advertised 


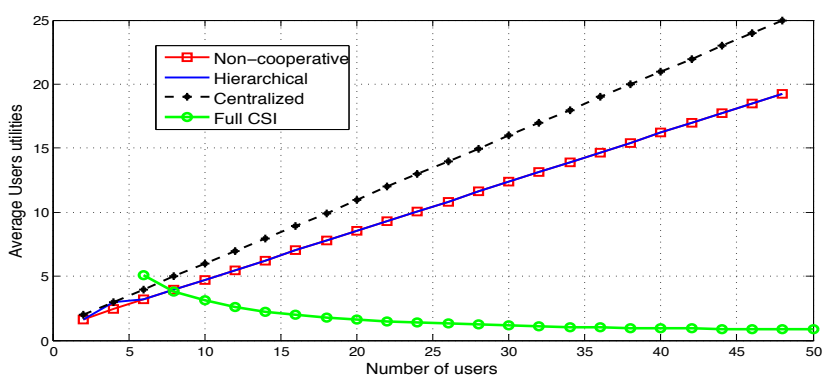

Fig. 6. Average users' utilities for increasing number of competing users.

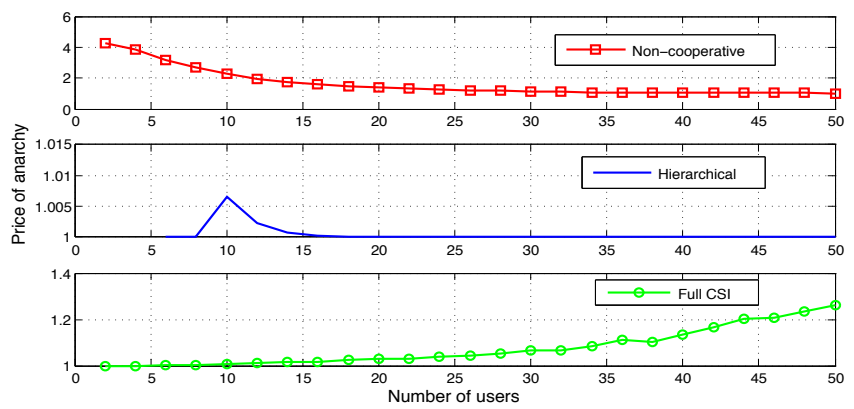

Fig. 7. The price of anarchy for increasing number of competing users $n$.

by the $\mathrm{MC}$ which results in the same outcome as for the non-cooperative case. Now, looking at the full CSI model, we remark that with a greater number of users, the users' utilities crucially decrease. This is justified by the fact that, when $n$ grows large, the MC becomes more saturated and users move over to the SC. As each new user means more saturation of the MC, users with worse channel conditions on the SC become interested in using the SC anyway which results in decreasing the users' utilities for the full CSI model. This contrasts with the situation when users compete for the equilibrium strategies they estimate (i.e., the hierarchical and the non-cooperative models) which show an increasing utility as the number of user grows.

3) Network utility: Let us now analyse the performance of the proposed approach on the network throughput at the equilibrium. To do so, we plot in Figure 7 the PoA for the different schemes studied. We observe several interesting phenomena here. First of all, for the full CSI model, increasing the numbers of users results both in a decrease in average throughput on both networks and in the overall use of the MC, which implies a higher PoA. Second, the PoA of the non-cooperative model is a decreasing function of the number of users. This result may seem surprising at first glance, as usually a bigger number of players means more anarchy. However, if we look at the objective function of the MC in Eq. (8), which is the expected throughput of the $\mathrm{MC}$, we clearly see that a bigger number of players is disadvantageous for the MC network which may get congested (since the probability that any of the players connected to the $\mathrm{MC}$ is active increases) and favorable for the SC which yet cannot. One important consequence here is that any mechanism aiming at stimulating the use of the $\mathrm{MC}$ network only through a small amount of proper signaling, should concentrate on doing it for smaller number of users. Finally, as we can see in Figure 7, the proposed hierarchical mechanism keeps the PoA almost equal to 1 even for a low number of users, and remains bounded above by the non-cooperative model in that case anyway. This suggests that introducing hierarchy in decision making between the different components of the network results in a better utilization of network and radio resources with respect to conventional centralized policies.

\section{CONCLUSION}

Our main goal within this paper is to design a dynamic offset which enables operators to steer traffic in order to provide a better user experience and enable the operator to move traffic from the cellular network. To do so, we have proposed a Bayesian hierarchical load-aware association method where partial asymmetric information from the $\mathrm{MC}$ and the SC are available in making optimal network selection decisions. The users' network selection decision is guided by CQI messages conveyed to the mobile users by the MC. Our main result is that we have shown that, in order to maximize its revenue, the network operator rather than increasing the throughput (which is costly and energy inefficient) - can drive the users to the MC network by a proper choice of information to transmit. We notably propose an algorithm for finding the dynamic offset for the Stackelberg model whose complexity is polynomial in the number of users. It turns out that the channel quality indicator the operator should choose is either very small or very large in comparison to the one maximizing the global throughput of both systems. We have also observed that the dynamic offset $\Psi$ at the Stackelberg equilibrium is decreasing with the number of active users $n$. This is to say that when $n$ grows, users can connect more often to the SC network without wasting the MC resources, which results in a smaller $\Psi$ above which users connect to SC. One important consequence is that any mechanism aiming at stimulating the use of the MC network only through a small amount of proper signaling, should concentrate on doing it for smaller number of users. Furthermore, we have observed that the PoA of the proposed hierarchical mechanism is almost equal to 1 even for a low number of users, and remains bounded above by the non-cooperative model as the number of users grows. Achieving these results offers hope that such a smart mechanism can be designed around SC integration in HetNets and can be implemented using the notion of Cell Selection Bias (CSB) proposed by LTE standards.

\section{REFERENCES}

[1] "Technical specification group radio access network; scenarios and requirements for small cell enhancements for e-utra and e-utran (release 12)," vol. 3GPP TR 36.932 V12.0.0, 2012. [Online]. Available: http://www.3gpp.org/ftp/Specs/archive/36_series/36.932/36932c00.zip

[2] J. G. Andrews, H. Claussen, M. Dohler, S. Rangan, and M. C. Reed, "Femtocells: Past, Present, and Future," IEEE Journal on Selected Areas in Communications, vol. 30, no. 3, pp. 497-508, Apr. 2012.

[3] A. Damnjanovic, J. Montojo, Y. Wei, T. Ji, T. Luo, M. Vajapeyam, T. Yoo, O. Song, and D. Malladi, "A survey on 3gpp heterogeneous networks," Wireless Communications, IEEE, vol. 18, no. 3, pp. 1021, 2011. 
[4] Ericsson, "LTE Release 12 - taking another step toward the Networked Society," White paper, Jan. 2013. [Online]. Available: http://goo.gl/BFMFR

[5] L. Wang and G.-S. Kuo, "Mathematical modeling for network selection in heterogeneous wireless networks: A tutorial," Communications Surveys Tutorials, IEEE, vol. 15, no. 1, pp. 271-292, 2013.

[6] S. Deb, P. Monogioudis, J. Miernik, and J. P. Seymour, "Algorithms for enhanced inter cell interference coordination (eicic) in lte hetnets," in IEEE/ACM Transactions on Networking, 2013.

[7] R. Mahindra, H. Viswanathan, K. Sundaresan, M. Y. Arslan, and S. Rangarajan, "A practical traffic management system for integrated lte-wifi networks," in ACM MobiCom, 2014.

[8] A. Ting, D. Chieng, K. H. Kwong, I. Andonovic, and K. Wong, "Dynamic backhaul sensitive network selection scheme in lte-wifi wireless hetnet," in IEEE PIMRC, Sept 2013.

[9] M. Haddad, S. E. Elayoubi, E. Altman, and Z. Altman, "A hybrid approach for radio resource management in heterogeneous cognitive networks," IEEE Journal on Selected Areas in Communications Special Issue on Advances in Cognitive Radio Networking and Communications, vol. 2, no. 6, pp. 733-741, April 2010.

[10] E. Aryafar, A. Keshavarz-Haddad, M. Wang, and M. Chiang, "Rat selection games in hetnets," in IEEE INFOCOM, 2013.

[11] J. G. Andrews, S. Singh, Q. Ye, X. Lin, and H. S. Dhillon, "An overview of load balancing in hetnets: Old myths and open problems," Wireless Communications, IEEE, vol. 21, no. 2, pp. 18-25, Apr. 2014.

[12] O. Semiari, W. Saad, S. Valentin, M. Bennis, and B. Maham, "Matching theory for priority-based cell association in the downlink of wireless small cell networks," in IEEE ICASSP, May 2014.

[13] L. Giupponi, R. Agusti, J. Perez-Romero, and O. Sallent, "Joint radio resource management algorithm for multi-RAT networks," in Global Telecommunications Conference (Globecom), IEEE, vol. 6, Dec 2005.

[14] E. Stevens-Navarro, Y. Lin, and V. W. S. Wong, "An MDP-Based Vertical Handoff Decision Algorithm for Heterogeneous Wireless Networks," IEEE Transactions on Vehicular Technology, vol. 57, no. 2, pp. 1243-1254, 2008

[15] D. Kumar, E. Altman, and J.-M. Kelif, "Globally Optimal UserNetwork Association in an 802.11 WLAN and 3G UMTS Hybrid Cell," in Proc. of 20th International Teletraffic Congress (ITC), Ottawa, Canada, June 2007.

[16] Nokia Solutions and Networks, "Multi-cell Radio Resource Management: centralized or decentralized?" White paper, Jan. 2014.

[17] L. Scalia, T. Biermann, C. Choi, K. Kozu, and W. Kellerer, "Powerefficient mobile backhaul design for comp support in future wireless access systems," in Computer Communications Workshops (INFOCOM WKSHPS), 2011 IEEE Conference on, 2011, pp. 253-258.

[18] F. Diehm and G. Fettweis, "On the impact of signaling delays on the performance of centralized scheduling for joint detection cooperative cellular systems," in IEEE WCNC, 2011, pp. 1897-1902.

[19] S. Singh, J. Andrews, and G. de Veciana, "Interference shaping for improved quality of experience for real-time video streaming," Selected Areas in Communications, IEEE Journal on, vol. 30, no. 7, pp. 1259-1269, 2012.

[20] D. Lopez-Perez, I. Guvenc, G. de la Roche, M. Kountouris, T. Quek, and J. Zhang, "Enhanced intercell interference coordination challenges in heterogeneous networks," Wireless Communications, IEEE, vol. 18, no. 3, pp. 22-30, June 2011.

[21] S. Singh, H. Dhillon, and J. Andrews, "Offloading in heterogeneous networks: Modeling, analysis, and design insights," Wireless Communications, IEEE Transactions on, vol. 12, no. 5, pp. 2484-2497, 2013.

[22] M. Simsek, M. Bennis, M. Debbah, and A. Czylwik, "Rethinking offload: How to intelligently combine wifi and small cells?" in Communications (ICC), 2013 IEEE International Conference on, June 2013.

[23] Q. Ye, B. Rong, Y. Chen, M. Al-Shalash, C. Caramanis, and J. Andrews, "User association for load balancing in heterogeneous cellular networks," Wireless Communications, IEEE Transactions on, vol. 12, no. 6, pp. 2706-2716, June 2013.

[24] M. Haddad, H. Sidi, P. Wiecek, and E. Altman, "Automated dynamic offset applied to cell association," in INFOCOM, Toronto, Canada, April 2014.

[25] E. Altman, P. Wiecek, and M. Haddad, "The association problem with misleading partial channel state information," in IEEE WCNC, Paris, France, May, 2012

[26] J. Li, A. Bose, and Y. Q. Zhao, "Rayleigh flat fading channels' capacity," in Proceedings of the 3rd Annual Communication Networks and Services Research Conference, May 2005.

[27] S. M. Perlaza, M. Debbah, S. Lasaulce, and H. Bogucka, "On the benefits of bandwidth limiting in decentralized vector multiple access channels," in CROWNCOM, Hannover, Germany, June, 2009.

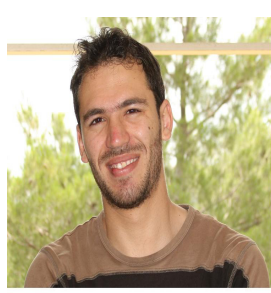

Majed Haddad is an assistant professor at the University of Avignon, France since 2014, where his main research interests are in radio resource management, heterogeneous networks, green networks, complex networks and game theory. In 2004, Majed received his electrical engineering diploma from the National Engineering School of Tunis (ENIT), Tunisia. He received the master degree from the university of Nice Sophia-Antipolis in France in 2005, and a doctorate in Electrical Engineering from Eurecom institute in 2008. In 2009, he joined France Telecom R\&D as a post-doctoral research fellow. In 2011, he joined the university of Avignon in France as a researcher assistant. From 2012 to 2014, Dr. Haddad was a research engineer at INRIA Sophia-Antipolis in France under an INRIA Alcatel-Lucent Bell Labs fellowship. Majed has published more than 50 research papers in international conferences, journals, book chapters and patents. He also acts as TPC chair, TPC member and reviewer for various prestigious conferences and journals.

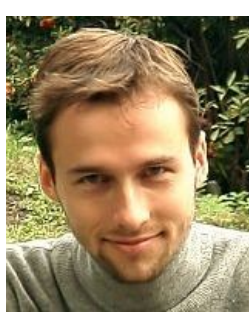

Piotr Więcek received the M.Sc. and Ph.D. degrees in mathematics from the Wroclaw University of Technology, Wroclaw, Poland, in 2000 and 2004, respectively. He is currently an assistant professor at the Faculty of Fundamental Problems of Technology, Wroclaw University of Technology. He specializes in stochastic optimization and dynamic game theory, and their applications in computer science and telecommunications. His main interests in telecommunications include heterogeneous networks, green networks and power control. He is a member of the executive board of International Society of Dynamic Games.

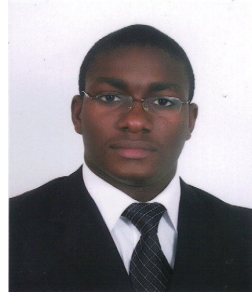

Habib B. A. Sidi received his Engineering degree in networks and mobile radio engineering in 2009 from the National Institute of Posts and Telecommunications (INPT), Morocco. He obtained the $\mathrm{PhD}$ degree from the computer science laboratory of University of Avignon (LIA), France, in 2012. He has spent two years as postdoctorate fellow successively at Orange Labs in 2013 and is currently associated with the DYOGENE team at INRIA in Paris. His research interests are on distributed computing, stochastic and online optimization, wireless networks engineering, network modelling and performance evaluation. He is interested also in System/MAC layer simulations and experimentations in mobile networks.

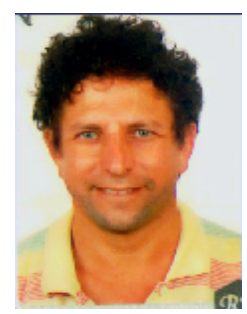

Eitan Altman received the B.Sc. degree in electrical engineering (1984), the B.A. degree in physics (1984) and the Ph.D. degree in electrical engineering (1990), all from the Technion-Israel Institute, Haifa. In (1990) he further received his B.Mus. degree in music composition in Tel-Aviv University. Since 1990, he has been with INRIA (National research institute in informatics and control) in Sophia-Antipolis, France. His current research interests include performance evaluation and control of telecommunication networks and in particular congestion control, wireless communications and networking games. He is in the editorial board of several scientific journals: JEDC, COMNET, DEDS and WICON. He has been the (co)chairman of the program committee of several international conferences and workshops on game theory, networking games and mobile networks. 